\title{
How Important is the Detection of Changes in Active Constraints in Real-Time Optimization? ${ }^{\star}$
}

\author{
Saurabh Deshpande* Bala Srinivasan ${ }^{* *}$ Dominique Bonvin* \\ * Laboratoire d'Automatique, École Polytechnique Fédérale de \\ Lausanne, CH-1015 Lausanne, Switzerland (e-mail: \\ \{saurabh.deshpande,dominique.bonvin\}@epfl.ch) \\ ** Department of Chemical Engineering, École Polytechnique Montréal, \\ Canada (e-mail: bala.srinivasan@polymtl.ca)
}

\begin{abstract}
In real-time optimization, enforcing the constraints that need to be active is important for optimality. In fact, it has been established in the context of parametric variations that, if these constraints are not satisfied, the optimality loss would be $O(\eta)-\eta$ denoting the magnitude of the parametric variations. In contrast, the loss of optimality upon enforcing the correct set of active constraints would be $O\left(\eta^{2}\right)$. However, no result is available when the set of active constraints changes due to parametric variations, which forms the subject of this paper. It is shown that, if the optimal solution is unique for each $\eta$, enforcing the constraints that are strictly active in the nominal solution will lead to $O\left(\eta^{2}\right)$ loss in optimality, even when the other active constraints of the perturbed system are different from that of the nominal system. This, in turn, means that, in any input adaptation scheme for real-time optimization, the detection of changes in active constraints is not important as long as it is possible to enforce the strictly active constraints of the nominal solution.
\end{abstract}

Keywords: Real-time optimization; static optimization; parametric nonlinear programs; strict active set; input adaptation; optimality loss.

\section{INTRODUCTION}

Optimal steady-state operation in the presence of uncertainty is an important objective in the process industries. The idea is to adapt the nominal optimal inputs, which are calculated using the available process model, to achieve optimality and feasibility of plant operation. Numerous real-time optimization (RTO) algorithms have been proposed in the literature (Marlin and Hrymak [1997], Gao and Engell [2005], Tatjewski [2008], Chachuat et al. [2009], Woodward et al. [2010]).

Since perfect adaptation may not be possible in practice, it becomes essential to be able to compare the performance of a given set of adapted inputs with that of the perturbed optimal inputs. This paper presents an analysis of the performance loss associated with input adaptation by focusing on the set of constraints that are kept active.

A few words are in order to explain the motivation behind the focus on active constraints. For static RTO problems modeled in terms of parametric nonlinear programs (NLP), in which uncertainty is described by parametric variations, the necessary conditions of optimality ( $\mathrm{NCO}$ ) include both constraint-seeking and sensitivity-seeking components. The former require that certain constraints are active, while the latter require that reduced gradients are forced to zero (Bazaraa et al. [1993]). Accordingly,

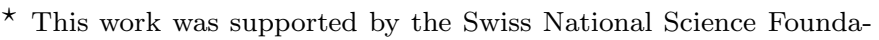
tion (SNSF) under Grant \#200020-113292.
}

two sets of directions in input space can be identified such that adaptation along directions in one set does not affect the active constraints, while adaptation along directions in the other set does (François et al. [2005]). Furthermore, it has been shown that input adaptation along the constraint-seeking directions has a larger effect on the cost than adaptation along the sensitivity-seeking directions (Chachuat et al. [2008]). Hence, if full adaptation is not possible, adaptation that favors meeting the active constraints should be preferred.

Note that the aforementioned arguments describe the situation only around the set of nominal optimal inputs. Another object of interest, especially important from the point of view of optimality, is the set of perturbed optimal inputs. Since an iterative RTO algorithm will typically start from the nominal solution, the resulting adapted inputs might share only certain features with the perturbed optimal solution. One such feature is the set of active constraints. Hence, the following question arises naturally: if different algorithms yield different adapted inputs, can the effect of these inputs on the cost be inferred from the features they share with the perturbed optimal inputs? In other words, it is essential to perform a joint analysis of the features of the nominal optimal inputs, the adapted inputs and the perturbed optimal inputs. This is the spirit of the present work, which investigates optimality loss due to different sets of adapted inputs that conserve some or other elements of the nominal active constraint set and 
thus share only a few features of the perturbed optimal solution.

This investigation will try to underscore the importance of the strict active set of constraints in designing RTO schemes for static optimization. More specifically, the following important result is proved under reasonable conditions: the optimality loss incurred by enforcing only the constraints that are strictly active in the nominal solution is only $\mathrm{O}\left(\eta^{2}\right)$, regardless of whether or not the optimal active set changes.

The paper is organized as follows. Section 2 gives the mathematical formulation of the parametric NLP, followed by two useful lemmas. Section 3 investigates the optimality loss associated with input adaptation for the case of small parametric variations. Section 4 presents an example that illustrates optimality loss as a function of the magnitude of the parametric variations. Finally, Section 5 summarizes the results and proposes some topics for future research.

\section{REAL-TIME OPTIMIZATION UNDER UNCERTAINTY}

\subsection{Problem Formulation and Optimality Conditions}

We consider the following parametric NLP problem:

$$
\begin{array}{cl}
\min _{\mathbf{u}} & J(\mathbf{u}, \boldsymbol{\theta}) \\
\text { s.t. } & G_{i}(\mathbf{u}, \boldsymbol{\theta}) \leq 0, \quad i=1, \ldots, n_{G}, \\
& \mathbf{u} \in \mathbb{R}^{n_{u}}, \quad \boldsymbol{\theta} \in \mathbb{R}^{n_{\theta}} .
\end{array}
$$

where $\mathbf{u}$ is the input vector, $J$ the cost function and $G_{i}$ the $i^{\text {th }}$ constraint. $J$ and all $G_{i}$ are assumed once continuously differentiable in all arguments. The nominal value of the parameters is $\boldsymbol{\theta}_{0}$.

We assume linear independence of the gradients of the active constraints at the nominal solution (constraint qualification) so that the Karush-Kuhn-Tucker NCO hold at the nominal optimal solution (Bazaraa et al. [1972], Peterson [1973]):

$$
\begin{aligned}
\frac{\partial J}{\partial \mathbf{u}}+\lambda^{T} \frac{\partial \mathbf{G}}{\partial \mathbf{u}} & =\mathbf{0}, \\
\lambda_{i} G_{i}(\mathbf{u}, \boldsymbol{\theta}) & =0, \quad i=1, \ldots, n_{G}, \\
\lambda_{i} \geq 0, & i=1, \ldots, n_{G} .
\end{aligned}
$$

Let the nominal solution $\left(\mathbf{u}^{*}, \boldsymbol{\lambda}^{*}\right)$ be such that:

$$
\begin{array}{lll}
\lambda_{i}^{*}>0 \quad \& \quad & G_{i}\left(\mathbf{u}^{*}, \boldsymbol{\theta}_{0}\right)=0, & i \in \mathcal{I}^{*}, \\
\lambda_{i}^{*}=0 \quad \& \quad & G_{i}\left(\mathbf{u}^{*}, \boldsymbol{\theta}_{0}\right)=0, & i \in \mathcal{J}^{*}, \\
\lambda_{i}^{*}=0 \quad \& \quad & G_{i}\left(\mathbf{u}^{*}, \boldsymbol{\theta}_{0}\right)<0, & i \in \mathcal{K}^{*}, \\
\mathcal{I}^{*} \cup \mathcal{J}^{*} \cup \mathcal{K}^{*}=\left\{1, \ldots, n_{G}\right\} . &
\end{array}
$$

In terms of the notation above, the constraint qualification means the independence of the column vectors of $\frac{\partial \mathbf{G}_{\mathcal{I}^{*} \cup \mathcal{J}^{*}}}{\partial \mathbf{u}}$.

In practice, knowledge of (3) is available via off-line numerical optimization. Note that there are no elements common in $\mathcal{I}^{*}, \mathcal{J}^{*}$ and $\mathcal{K}^{*}$. Also, define $\mathcal{A}^{*}=\mathcal{I}^{*} \cup \mathcal{J}^{*} . \mathcal{A}^{*}$ is the active constraint set, $\mathcal{I}^{*}$ the strictly active constraint set, $\mathcal{J}^{*}$ the marginally active set, and $\mathcal{K}^{*}$ the inactive set.
The main difference between the two parts of the active set is that non-satisfaction of the strictly active constraints $\mathcal{I}^{*}$ would cause a more significant impact on the cost function than non-satisfaction of the marginally active constraints $\mathcal{J}^{*}$. This aspect will be quantified later.

\subsection{Uncertainty Description}

The following type of parametric variations is considered:

$$
\tilde{\boldsymbol{\theta}}=\boldsymbol{\theta}_{0}+\eta \boldsymbol{\xi}^{\boldsymbol{\theta}}, \quad \eta \in \mathcal{B}_{\eta}
$$

where $\boldsymbol{\xi}^{\boldsymbol{\theta}}$ is a vector - of unit Euclidean norm - in the parameter space $\mathbb{R}^{n_{\theta}}$, and $\mathcal{B}_{\eta}$ is a small ball around zero.

Let $\tilde{\mathbf{u}}$ denote the perturbed optimal inputs. Henceforth, we will assume that the perturbed optimal solution $(\tilde{\mathbf{u}}, \tilde{\boldsymbol{\lambda}})$ also satisfies the NCO (2) for each $\eta$. Hence, the perturbed optimal solution for a given $\eta$ satisfies:

$$
\begin{array}{llll}
\tilde{\lambda}_{i}>0 & \& & G_{i}(\tilde{\mathbf{u}}, \tilde{\boldsymbol{\theta}})<0, & i \in \tilde{\mathcal{I}}, \\
\tilde{\lambda}_{i}=0 & \& & G_{i}(\tilde{\mathbf{u}}, \tilde{\boldsymbol{\theta}})<0, & i \in \tilde{\mathcal{J}} \\
\tilde{\lambda}_{i}=0 & \& & G_{i}(\tilde{\mathbf{u}}, \tilde{\boldsymbol{\theta}})=0, & i \in \tilde{\mathcal{K}} \\
\tilde{\mathcal{I}} \cup \tilde{\mathcal{J}} \cup \tilde{\mathcal{K}}=\left\{1, \ldots, n_{G}\right\} . &
\end{array}
$$

The index sets $\tilde{\mathcal{I}}, \tilde{\mathcal{J}}$ and $\tilde{\mathcal{K}}$ can, in general, be different from $\mathcal{I}^{*}, \mathcal{J}^{*}$ and $\mathcal{K}^{*}$. Without loss of generality, suppose that there is a change in active set around $\eta=0$, with three possible active sets: At $\eta=0$, the strictly active, marginally active and inactive sets correspond to $\mathcal{I}^{*}, \mathcal{J}^{*}$, and $\mathcal{K}^{*}$, respectively; at $\eta=0_{+}$, these sets are $\tilde{\mathcal{I}}_{+}, \tilde{\mathcal{J}}_{+}$, and $\tilde{\mathcal{K}}_{+}$, while at $\eta=0_{-}$, these sets are $\tilde{\mathcal{I}}_{-}, \tilde{\mathcal{J}}_{-}$, and $\tilde{\mathcal{K}}_{-}$. The relationship between them is studied next.

Toward this end, three lemmas are introduced to show that, if the solution is unique, then the inputs and the Lagrange multipliers are indeed continuous. The proofs are presented in the Appendix A.

Lemma 1. (Continuity of $\tilde{\mathbf{u}}$ with respect to $\eta$ ). Consider the NLP problem (1) with the cost and constraints being differentiable with respect to the inputs and parameters. If $\tilde{\mathbf{u}}(\eta)$ is unique for all $\eta \in \mathcal{B}_{\eta}$, then it is continuous with respect to $\eta$. Hence, $\tilde{\mathbf{u}}(\eta)-\mathbf{u}^{*}=O(\eta)$.

Lemma 2. (Relation between $\mathcal{I}^{*}, \tilde{\mathcal{I}}$ and $\mathcal{J}^{*}$ ).

Consider the NLP problem (1) with the cost and constraints being differentiable with respect to the inputs and parameters. If Lemma 1 holds, then $\mathcal{I}^{*} \subseteq \tilde{\mathcal{I}}$. Further, $\tilde{\mathcal{I}} \backslash \mathcal{I}^{*} \subseteq \mathcal{J}^{*}$.

Lemma 2 essentially states that, due to the continuity of optimal inputs, the strict active set will still remain strictly active, and the inactive set will remain inactive. Only the elements in the marginally active set can change sides, i.e., they can either stay marginally active, become strictly active or become inactive.

Hence, we can write:

$$
\mathcal{I}^{*} \subseteq \tilde{\mathcal{I}}, \quad \mathcal{K}^{*} \subseteq \tilde{\mathcal{K}}, \quad \tilde{\mathcal{J}} \subseteq \mathcal{J}^{*}, \quad \tilde{\mathcal{A}} \subseteq \mathcal{A}^{*}
$$

Note that $\mathcal{I}^{*}$ is the minimal strict active set, i.e. smaller than or equal to $\tilde{\mathcal{I}}_{+}$and $\tilde{\mathcal{I}}_{-}$, while $\mathcal{A}^{*}$ is the maximal active set, i.e., larger than or equal to $\tilde{\mathcal{A}}_{+}$and $\tilde{\mathcal{A}}_{-}$.

Lemma 3. (Continuity of $\tilde{\boldsymbol{\lambda}}$ with respect to $\eta$ ). Consider the NLP problem (1) with the cost and constraints being 
differentiable with respect to the inputs and parameters. If Lemma 1 holds, then the adjoint variables $\tilde{\boldsymbol{\lambda}}(\eta)$ are continuous with respect to $\eta$. Hence, $\tilde{\boldsymbol{\lambda}}(\eta)-\boldsymbol{\lambda}^{*}=O(\eta)$.

In summary, there are no discontinuities in the optimal solution, and the Lagrange multipliers and thus the optimal cost and optimal constraint functions are also continuous in $\eta$.

\subsection{Optimality Loss}

Definition 4. (Optimality Loss). For parametric variations given by (4), the difference between the cost resulting from a given set of inputs $\mathbf{u}$ and the perturbed optimal cost is called optimality loss and is denoted by $\delta J$ :

$$
\delta J(\mathbf{u}):=J(\mathbf{u}, \tilde{\boldsymbol{\theta}})-J(\tilde{\mathbf{u}}, \tilde{\boldsymbol{\theta}}),
$$

where $\tilde{\mathbf{u}}$ denotes the perturbed optimal inputs.

Theorem 5. Consider the NLP problem (1) with the cost and constraints being differentiable with respect to the inputs and parameters. If no adaptation is done, the optimality loss is $O(\eta)$.

\section{Proof:}

Using the differentiability properties of $J$ with respect to $\mathbf{u}$, it is possible to consider the first-order Taylor series expansion of $J(\mathbf{u}, \tilde{\boldsymbol{\theta}})$ around $(\tilde{\mathbf{u}}, \tilde{\boldsymbol{\theta}})$ :

$$
\delta J(\mathbf{u})=\frac{\partial J}{\partial \mathbf{u}}(\tilde{\mathbf{u}}, \tilde{\boldsymbol{\theta}})\{\mathbf{u}-\tilde{\mathbf{u}}\}+O\left(\{\mathbf{u}-\tilde{\mathbf{u}}\}^{2}\right) .
$$

From (2) and (5), one can write:

$$
\frac{\partial J}{\partial \mathbf{u}}(\tilde{\mathbf{u}}, \tilde{\boldsymbol{\theta}})+\tilde{\boldsymbol{\lambda}}_{\tilde{\mathcal{I}}}^{T} \frac{\partial \mathbf{G}_{\tilde{\mathcal{I}}}}{\partial \mathbf{u}}(\tilde{\mathbf{u}}, \tilde{\boldsymbol{\theta}})=\mathbf{0} .
$$

Using (9) in (8), gives:

$$
\delta J(\mathbf{u})=-\tilde{\boldsymbol{\lambda}}_{\tilde{\mathcal{I}}}^{T} \frac{\partial \mathbf{G}_{\tilde{\mathcal{I}}}}{\partial \mathbf{u}}(\tilde{\mathbf{u}}, \tilde{\boldsymbol{\theta}})\{\mathbf{u}-\tilde{\mathbf{u}}\}+O\left(\{\mathbf{u}-\tilde{\mathbf{u}}\}^{2}\right) .
$$

When the nominal optimal inputs are applied, Lemma 1 says that $\mathbf{u}^{*}-\tilde{\mathbf{u}}=O(\eta)$, which implies $\delta J\left(\mathbf{u}^{*}\right)=O(\eta)$.

\section{OPTIMALITY LOSS WHEN ACTIVE CONSTRAINTS ARE MET}

This section investigates the optimality loss when the nominal active constraints are kept active using input adaptation.

\subsection{Optimality Loss with Same Optimal Active Set}

The simplest case is when there is no change in the active constraints, i.e. $\mathcal{I}^{*}=\tilde{\mathcal{I}}_{+}=\tilde{\mathcal{I}}_{-}, \mathcal{J}^{*}=\tilde{\mathcal{J}}_{+}=\tilde{\mathcal{J}}_{-}$, and $\mathcal{K}^{*}=\tilde{\mathcal{K}}_{+}=\tilde{\mathcal{K}}_{-}$

We consider adapting the inputs so as to conserve the active sets. Let $\hat{\mathbf{u}}=\mathbf{u}^{*}+\eta \boldsymbol{\xi}^{u}$ represent the adapted inputs that keep the set $\mathcal{A}^{*}$ active without violating any other constraint, i.e., they satisfy the following conditions for the perturbed system: ${ }^{1}$

\footnotetext{
1 Note that the value of $\eta$ used for constructing $\hat{\mathbf{u}}$ is the same as the magnitude of parametric variations in (4).
}

$$
\begin{array}{ll}
G_{i}(\hat{\mathbf{u}}, \tilde{\boldsymbol{\theta}})=0, & \forall i \in \mathcal{I}^{*}, \\
G_{i}(\hat{\mathbf{u}}, \tilde{\boldsymbol{\theta}})=0, & \forall i \in \mathcal{J}^{*}, \\
G_{i}(\hat{\mathbf{u}}, \tilde{\boldsymbol{\theta}}) \ngtr 0, & \forall i \in \mathcal{K}^{*} .
\end{array}
$$

We assume that, for the given NLP (1) and $\tilde{\boldsymbol{\theta}}$ under consideration, there exist solution(s) to the system of equations (11).

Theorem 6. If the perturbed optimal solution $\tilde{\mathbf{u}}(\eta)$ is unique for each $\eta$ and the active set does not change, i.e. $\mathcal{A}^{*}=\tilde{\mathcal{A}}$, then the optimality loss associated with an input adaptation that keeps the constraints $\mathcal{A}^{*}$ active is $O\left(\eta^{2}\right)$.

Proof: Using the Taylor series expansion of $\mathbf{G}_{\tilde{\mathcal{I}}}(\hat{\mathbf{u}}, \tilde{\boldsymbol{\theta}})$ around $(\tilde{\mathbf{u}}, \tilde{\boldsymbol{\theta}})$ yields:

$\mathbf{G}_{\tilde{\mathcal{I}}}(\hat{\mathbf{u}}, \tilde{\boldsymbol{\theta}})=\mathbf{G}_{\tilde{\mathcal{I}}}(\tilde{\mathbf{u}}, \tilde{\boldsymbol{\theta}})+\frac{\partial \mathbf{G}_{\tilde{\mathcal{I}}}}{\partial \mathbf{u}}(\tilde{\mathbf{u}}, \tilde{\boldsymbol{\theta}})\{\hat{\mathbf{u}}-\tilde{\mathbf{u}}\}+O\left(\{\hat{\mathbf{u}}-\tilde{\mathbf{u}}\}^{2}\right)$.

Note that $\mathbf{G}_{\tilde{\mathcal{I}}}(\hat{\mathbf{u}}, \tilde{\boldsymbol{\theta}})=0$ by the definition of $\hat{\mathbf{u}}$ since $\tilde{\mathcal{I}}=\mathcal{I}^{*}$. Also, $\mathbf{G}_{\tilde{\mathcal{I}}}(\tilde{\mathbf{u}}, \tilde{\boldsymbol{\theta}})=0$ by definition of the active set of the perturbed optimum. Since $\hat{\mathbf{u}}-\mathbf{u}^{*}=O(\eta)$ by construction and $\tilde{\mathbf{u}}-\mathbf{u}^{*}=O(\eta)$ from Lemma $1, \hat{\mathbf{u}}-\tilde{\mathbf{u}}=O(\eta)$. Using all these facts, (12) becomes:

$$
\frac{\partial \mathbf{G}_{\tilde{\mathcal{I}}}}{\partial \mathbf{u}}(\tilde{\mathbf{u}}, \tilde{\boldsymbol{\theta}})\{\hat{\mathbf{u}}-\tilde{\mathbf{u}}\}=O\left(\eta^{2}\right),
$$

which, when combined with (10), leads to $\delta J(\hat{\mathbf{u}})=$ $O\left(\eta^{2}\right)$.

Remark: Note that keeping $\mathbf{G}_{\mathcal{J}^{*}}(\hat{\mathbf{u}}, \tilde{\boldsymbol{\theta}})=0$ does not help toward optimality. This makes sense as the Lagrange multipliers corresponding to these constraints are zero, i.e., these constraints though active do not contribute to the cost. Hence, they could indeed be relaxed to become inactive.

\subsection{Optimality Loss with Change in Optimal Active Set}

For this scenario, several possibilities can be considered. One could use input adaptation to keep either $\mathcal{A}^{*}$, or $\tilde{\mathcal{A}}_{+}$ or $\tilde{\mathcal{A}}_{-}$, active over the ball $\mathcal{B}_{\eta}$. Also, as noted in the remark above, it is sufficient to keep the smallest of these sets, namely $\mathcal{I}^{*}$ or equivalently $\tilde{\mathcal{I}}_{+}$or $\tilde{\mathcal{I}}_{-}$, active. Of course, in all these adaptation strategies it is assumed that feasibility of the other constraints is guaranteed.

The next result proves that all these strategies are equivalent.

Theorem \%. Let the perturbed optimal solution $\tilde{\mathbf{u}}(\eta)$ be unique for each $\eta$. Consider Strategy (i) that adapts the input to keep the constraints $\mathcal{A}^{*}$ active, Strategy (ii) that keeps constraints $\tilde{\mathcal{A}}_{+}$active, Strategy (iii) that keeps con-

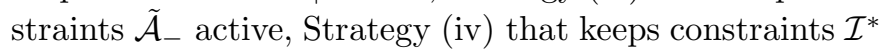
active, Strategy (v) that keeps constraints $\tilde{\mathcal{I}}_{+}$active and Strategy (vi) that keeps constraints $\tilde{\mathcal{I}}_{-}$active. The optimality loss associated with all these adaptation strategies is $O\left(\eta^{2}\right)$.

\section{Proof:}

Let $\mathcal{S}$ be the set of constraints enforced by the adopted strategy under consideration. It follows from (10) that the constraint enforcement of interest from the point of view 
of optimality loss is only $\mathcal{S} \cap \tilde{\mathcal{I}}$. Let us denote $\mathcal{C}=\mathcal{S} \cap \tilde{\mathcal{I}}$. Though other constraints are enforced, they do not have a first-order influence on the cost. Also note that all of the adopted strategies ensure that $\mathcal{I}^{*} \subseteq \mathcal{C}$.

On the other hand, there are other elements in $\tilde{\mathcal{I}}$ that are not enforced to zero. Let us denote them by $\mathcal{D}=\tilde{\mathcal{I}} \backslash \mathcal{C}$. Note that $\mathcal{D}$ does not contain any elements of $\mathcal{I}^{*}$.

Hence (10) can be written as

$$
\begin{aligned}
\delta J(\hat{\mathbf{u}})= & -\tilde{\boldsymbol{\lambda}}_{\mathcal{C}}^{T} \frac{\partial \mathbf{G}_{\mathcal{C}}}{\partial \mathbf{u}}(\tilde{\mathbf{u}}, \tilde{\boldsymbol{\theta}})\{\hat{\mathbf{u}}-\tilde{\mathbf{u}}\} \\
& -\tilde{\boldsymbol{\lambda}}_{\mathcal{D}}^{T} \frac{\partial \mathbf{G}_{\mathcal{D}}}{\partial \mathbf{u}}(\tilde{\mathbf{u}}, \tilde{\boldsymbol{\theta}})\{\hat{\mathbf{u}}-\tilde{\mathbf{u}}\}+O\left(\eta^{2}\right)
\end{aligned}
$$

Following the same arguments as in the proof of Theorem 6 , the first term of the expression can be shown to be $O\left(\eta^{2}\right)$.

From Lemma $3, \tilde{\boldsymbol{\lambda}}_{\mathcal{D}}-\boldsymbol{\lambda}_{\mathcal{D}}^{*}=O(\eta)$. However, since $\mathcal{D}$ does not contain any elements of $\mathcal{I}^{*}, \boldsymbol{\lambda}_{\mathcal{D}}^{*}=0$. This leads to $\tilde{\boldsymbol{\lambda}}_{\mathcal{D}}=O(\eta)$. Also, $(\hat{\mathbf{u}}-\tilde{\mathbf{u}})=O(\eta)$ as discussed in the proof of Theorem 6 , and so (14) becomes:

$$
\delta J(\hat{\mathbf{u}})=O\left(\eta^{2}\right)+O(\eta) \frac{\partial \mathbf{G}_{\mathcal{D}}}{\partial \mathbf{u}}(\tilde{\mathbf{u}}, \tilde{\boldsymbol{\theta}}) O(\eta)+O\left(\eta^{2}\right)=O\left(\eta^{2}\right)
$$

Remark: As noted earlier, $\mathcal{I}^{*}$ is the minimal of the sets characterizing the six strategies under consideration in Theorem 7. The implication of all six strategies being equal in terms of optimality loss is the following: $\mathcal{I}^{*}$ is the constraint set that needs to be kept active under parametric variations, even when the perturbed active set is different from the nominal one, to guarantee an optimality loss of no more than $\mathrm{O}\left(\eta^{2}\right)$.

In summary, when there is a unique perturbed optimal solution $\tilde{\mathbf{u}}(\eta)$ for each $\eta$, the optimality loss associated with keeping the nominal active set is always $O\left(\eta^{2}\right)$, irrespective of whether the active set for the plant, which in practice is unknown, is the same as that of the model or not. The practical implication of this result is that, at least locally, static RTO methods are better off in terms of cost by simply striving to maintain the active set found with the nominal model. As long as the adapted solution remains feasible, there is no need for any mechanism to detect which constraints become active from inactive and vice versa. ${ }^{2}$ On the other hand, failure to maintain the active set in RTO will result in an optimality loss of the order of $O(\eta)$.

\section{ILLUSTRATIVE EXAMPLE}

The following parametric NLP from Chachuat et al. [2008] is considered:

\footnotetext{
2 Note, however, that such a mechanism might be necessary to ensure feasibility.
}

$$
\begin{array}{cl}
\min _{\mathbf{u}} & J(\mathbf{u}, \boldsymbol{\theta})=\left(u_{1}-\theta_{1}\right)^{2}+\left(u_{2}-\theta_{1}\right)^{2}, \\
\text { s.t. } & G_{1}=u_{1}-\theta_{2}\left(1-u_{2}\right) \leq 0 \\
& G_{2}=u_{2} \theta_{3}-2\left(1-u_{1}\right) \leq 0 \\
& G_{3}=u_{1} \geq 0 \\
& G_{4}=u_{2} \geq 0 .
\end{array}
$$

Two different values of the nominal parameters $\boldsymbol{\theta}_{0}$ will be chosen to illustrate the results of two of the possible scenarios mentioned in Section 3, namely no change in optimal active set and decrease in the number of active constraints after parametric variations. It is possible to use different methods such as modifier adaptation (Marchetti et al. [2009]) or constraint control (Maarleveld and Rijnsdorp [1970], Marchetti et al. [2008], Woodward et al. [2010]) to keep the nominal active set active. This work uses the modifier-adaptation method.

\subsection{No Change in Active Set}

Let $\boldsymbol{\theta}_{0}=\left[\begin{array}{lll}0.75 & 1.4 & 1\end{array}\right]^{T}$ and the direction of parametric variation be $\boldsymbol{\xi}^{\boldsymbol{\theta}}=\frac{1}{\sqrt{3}}\left[\begin{array}{lll}1 & 1 & 1\end{array}\right]$. Let the range of uncertainty be quantified by $\mathcal{B}_{\eta}=[-0.05,0.05]$.

The nominal optimal solution is $\left(u_{1}^{*}, u_{2}^{*}\right)=$ $(0.6149,0.5608)$. Figure 1 plots the iso-cost contours for $\eta=-0.01$, the nominal optimal solution, the adapted inputs generated by modifier adaptation that conserve the nominal active set and the perturbed optimal inputs. The nominal optimal inputs are on the $G_{1}\left(\mathbf{u}, \boldsymbol{\theta}_{0}\right)=0$ curve, whereas the perturbed optimal inputs are on the $G_{1}(\mathbf{u}, \tilde{\boldsymbol{\theta}})=0$ curve. Hence, the active set remains unchanged under parametric variations. The same is verified for all $\eta \in \mathcal{B}_{\eta}$.

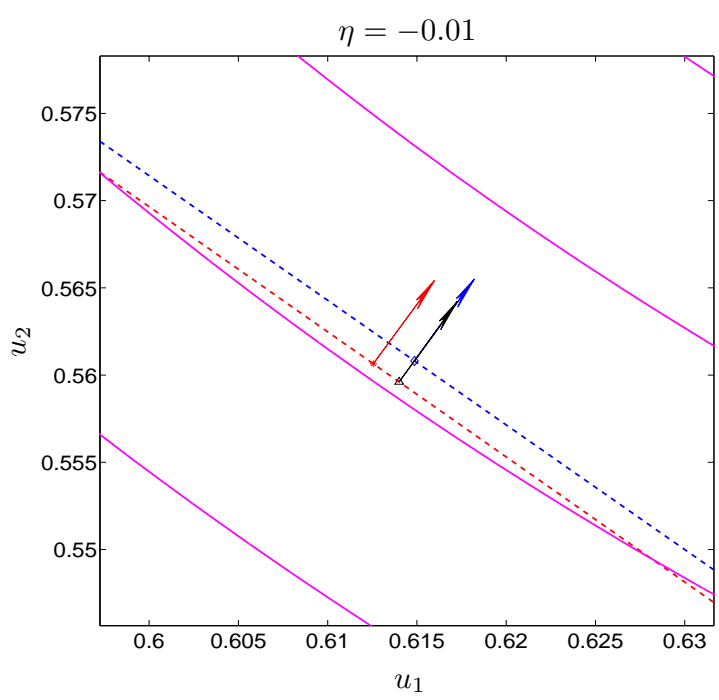

Fig. 1. Iso-cost contours for $\eta=-0.01$ depicting the nominal optimal inputs, the perturbed optimal inputs, and the adapted inputs. Refer to Appendix B for legend.

Figure 2 shows the optimality loss associated with input adaptation as a function of $\eta$ (Theorem 6 ). The $\mathrm{O}\left(\eta^{2}\right)$ fit of the plot agrees with the result of Theorem 6 . 


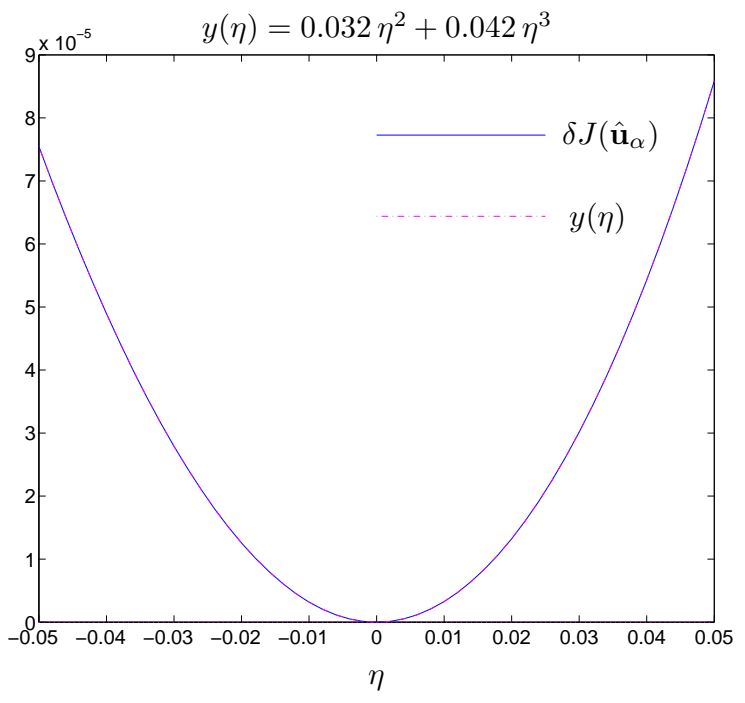

Fig. 2. Optimality loss associated with input adaptation that keeps the nominal active set active.

\subsection{Smaller Strict Active Set}

Let $\boldsymbol{\theta}_{0}=\left[\begin{array}{lll}1.517 & 1.417 & 1.017\end{array}\right]^{T}$ and the direction of parametric variation be $\boldsymbol{\xi}^{\boldsymbol{\theta}}=\frac{1}{\sqrt{3}}\left[\begin{array}{lll}1 & 1 & 1\end{array}\right]$. Let the range of uncertainty be quantified by $\mathcal{B}_{\eta}=[-0.005,0]$.

The nominal optimal solution is $\left(u_{1}^{*}, u_{2}^{*}\right)=$ $(0.7666,0.4590)$. For $\eta \leq-0.0032$, the constraint $G_{2}$ is no longer active, and the strict active set is smaller.

Figure 3 shows the iso-cost contours for $\eta=-0.026$. The nominal optimal inputs are at the intersection of the $G_{1}\left(\mathbf{u}, \boldsymbol{\theta}_{0}\right)=0$ and $G_{2}\left(\mathbf{u}, \boldsymbol{\theta}_{0}\right)=0$ curves and, similarly, the perturbed optimal inputs are at the intersection of the $G_{1}(\mathbf{u}, \tilde{\boldsymbol{\theta}})=0$ and $G_{2}(\mathbf{u}, \tilde{\boldsymbol{\theta}})=0$ curves.

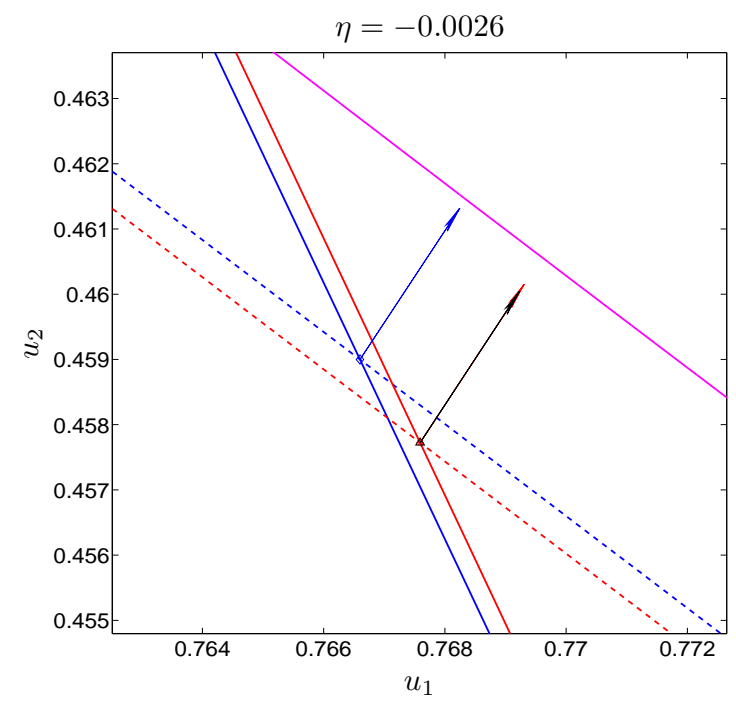

Fig. 3. Iso-cost contours for $\eta=-0.0026$ depicting the nominal optimal inputs, the perturbed optimal inputs and the adapted inputs. Refer to Appendix B for legend.
Figure 4 shows the iso-cost contours for $\eta=-0.004$. The perturbed optimal inputs are only on the $G_{1}(\mathbf{u}, \tilde{\boldsymbol{\theta}})=0$ curve.

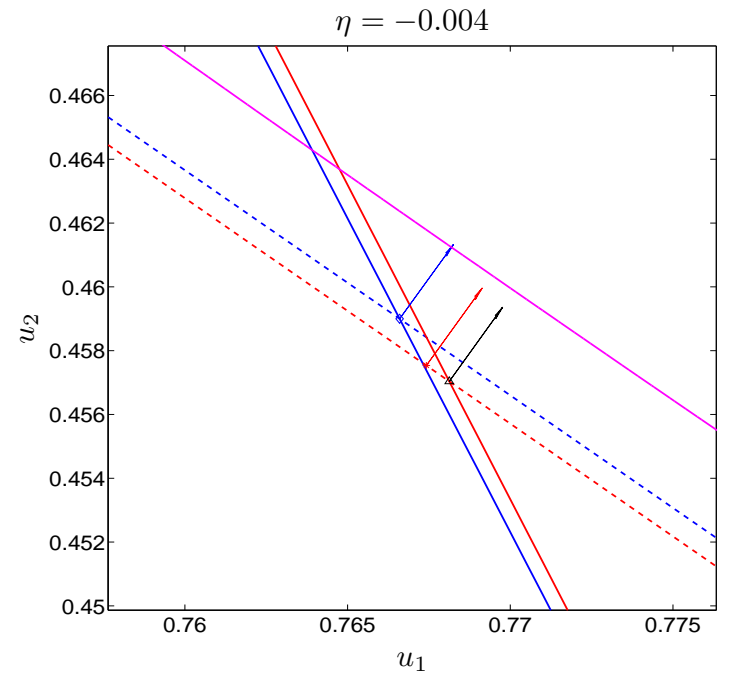

Fig. 4. Iso-cost contours for $\eta=-0.004$ depicting the nominal optimal inputs, the perturbed optimal inputs and the adapted inputs. Refer to Appendix B for legend.

On both contour plots, the adapted inputs generated using modifier adaptation lie at the intersection of the $G_{1}(\mathbf{u}, \tilde{\boldsymbol{\theta}})=0$ and $G_{2}(\mathbf{u}, \tilde{\boldsymbol{\theta}})=0$ curves. Thus, the adapted inputs keep the nominal active constraints active after parametric variations.

Finally, Figure 5 shows the optimality loss associated with input adaptation that conserves the nominal active set as a function of $\eta$ (Theorem 7). Note that the adapted inputs generated using modifier adaptation method coincide with the perturbed optimal solution for $\eta>-0.0032$.

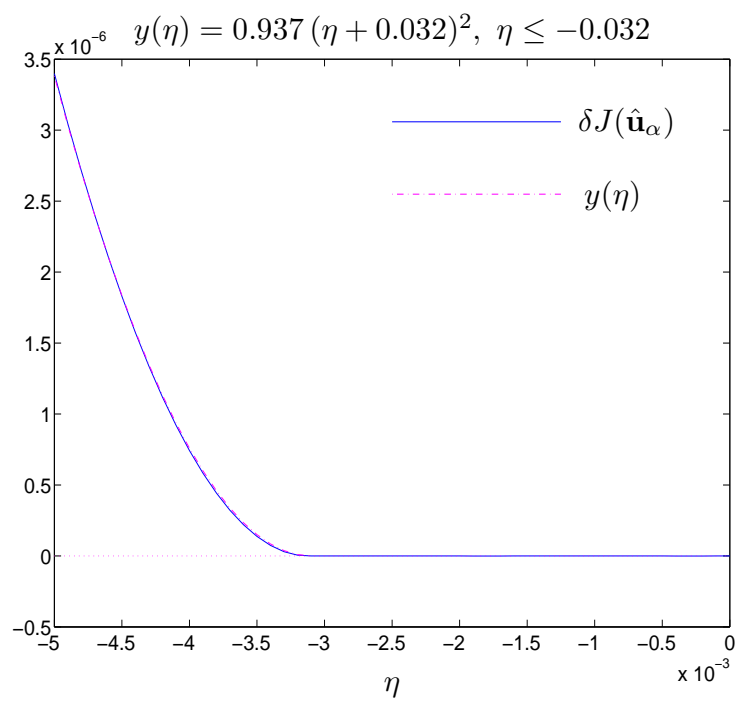

Fig. 5. Optimality loss associated with input adaptation that conserves the nominal active set. 


\section{CONCLUSIONS}

Input adaptation methods have become the cornerstone of static RTO. Any analysis of the performance of input adaptation methods must be based on the (properties of the) set of perturbed optimal inputs.

We have studied input adaptation strategies that counter parametric variations by keeping the nominal active set active, while still being feasible. For small parametric variations, the difference between the cost associated with adaptation and the perturbed optimal cost can be quantized as a function of $\eta$, the magnitude of the parametric variations. Under conditions that are standard for parametric NLP, the following important result has been proved: the optimality loss associated with adaptation that keeps the nominal active set is $O\left(\eta^{2}\right)$, even when there is a change in the set of active constraints. In addition, it is shown that conservation only of the minimal strict nominal active set $\left(\mathcal{I}^{*}\right)$ is what really matters to limit the optimality loss to order of $\mathrm{O}\left(\eta^{2}\right)$.

The practical implication of this result is that static RTO methods are sub-optimal by order of only $O\left(\eta^{2}\right)$ by simply striving to maintain the nominally active set. On the other hand, failure to maintain the strict active set in an RTO will certainly result in a larger optimality loss - at least of the order of $O(\eta)$

A limitation of the present results is the assumption of unique $\tilde{\mathbf{u}}(\eta)$ for each $\eta$. There exist examples of NLPs of type (1) for which this assumption does not hold. It is envisaged to extend the present analysis to such pathological cases in future research.

It is hoped that the results presented here will help analyze and compare the performance of existing static RTO methods and will also inspire the design of additional RTO schemes.

\section{REFERENCES}

M. Bazaraa, J. Goode, and C. Shetty. Constraint Qualifications Revisited. Management Science, 18(9):567 $573,1972$.

M. S. Bazaraa, H. D. Sherali, and C. M. Shetty. Nonlinear Programming: Theory and Algorithms. John Wiley and Sons, New York, 2nd edition, 1993.

C. Berge. Topological Spaces. Oliver \& Boyd Ltd., 1963.

B. Chachuat, A. Marchetti, and D. Bonvin. Process Optimization via Constraints Adaptation. J Process Contr, 18(3-4):244-257, 2008.

B. Chachuat, B. Srinivasan, and D. Bonvin. Adaptation Strategies for Real-Time Optimization. Comp. Chem. Eng., 33(10):1557-1567, 2009.

G. François, B. Srinivasan, and D. Bonvin. Use of Measurements for Enforcing the Necessary Conditions of Optimality in the Presence of Constraints and Uncertainty. J Process Contr, 15(6):701-712, 2005.

W. Gao and S. Engell. Iterative Set-Point Optimization of Batch Chromatography. Comp. Chem. Eng., 29:14011409, 2005.

A. Maarleveld and J. Rijnsdorp. Constraint Control on Distillation Columns. Automatica, 6:51-58, 1970.

A. Marchetti, B. Chachuat, and D. Bonvin. Real-Time Optimization via Adaptation and Control of the Con- straints. In 18th European Symposium on Computer Aided Process Engineering - ESCAPE 18, 2008.

A. Marchetti, B. Chachuat, and D. Bonvin. ModifierAdaptation Methodology for Real-Time Optimization. Ind. Eng. Chem. Res., 48(13):6022-6033, 2009.

T. E. Marlin and A. N. Hrymak. Real-Time Operations Optimization of Continuous Processes. In AIChE Symposium Series - CPC-V, volume 93, pages 156-164, 1997.

D. Peterson. A Review of Constraint Qualifications in Finite-Dimensional Spaces. SIAM Review, 15(3):639$654,1973$.

S. Robinson and R. Day. A Sufficient Condition for Continuity of Optimal Sets in Mathematical Programming. J. Math. Anal. \& Appl., 45:506 - 511, 1974.

P. Tatjewski. Advanced Control and On-line Process Optimization in Multilayer Structures. Annual Reviews in Control, 32:71-85, 2008.

L. Woodward, M. Perrier, and B. Srinivasan. Real-Time Optimization using a Jamming-Free Switching Logic for Gradient Projection on Active Constraints. Comp. \& Chem. Eng., 34(11):1863-1872, 2010.

\section{Appendix A. PROOF OF LEMMAS}

Proof of Lemma 1: Due to the differentiability assumptions (with respect to $\mathbf{u}$ and $\boldsymbol{\theta}$ ) on $J$ and $G_{i}$, Theorem 1 in Robinson and Day [1974] implies that the solution set $\{\tilde{\mathbf{u}}(\eta)\}$ of the parametric NLP (1) is upper semicontinuous, despite changes in the active set. Since $\tilde{\mathbf{u}}(\eta)$ is unique for each $\eta$ by assumption, the solution set $\{\tilde{\mathbf{u}}(\eta)\}$ is a singleton. Since upper semi-continuity and continuity coincide when the solution mapping is a singleton (Berge $[1963]), \tilde{\mathbf{u}}(\eta)$ is continuous with respect to $\eta$. Hence, we have $\tilde{\mathbf{u}}(\eta)-\mathbf{u}^{*}=O(\eta)$.

Proof of Lemma 2:

We will prove the result for $\eta=0_{+}$in the following three steps:

Step 1: (Impossibility of Inactive Constraints becoming Strictly Active)

First, it is easy to observe that no element of $\mathcal{K}^{*}$ can switch to $\tilde{\mathcal{I}}_{+}$. To see this, let us assume the contrary, namely, that there exists an index $k \in \tilde{\mathcal{I}}_{+} \cap \mathcal{K}^{*}$. Hence,

$$
G_{k}\left(\mathbf{u}^{*}, \boldsymbol{\theta}_{0}\right) \neq 0 ; \quad \text { but } G_{k}\left(\tilde{\mathbf{u}}\left(0_{+}\right), \tilde{\boldsymbol{\theta}}\left(0_{+}\right)\right)=0 .
$$

The last statement, however, is a contradiction since Lemma 1 holds and $G_{k}$ is continuous in both its arguments by assumption. Hence, it must be that $\tilde{\mathcal{I}}_{+} \cap \mathcal{K}^{*}=\emptyset$.

Step 2: (Impossibility of Decrease in Strict Active Set) Let us assume the contrary, viz., $\mathcal{I}^{*} \supset \tilde{\mathcal{I}}_{+}$. Hence, $\mathcal{I}^{*}=$ $\tilde{\mathcal{I}}_{+} \cup \mathcal{Z}$, for some index set $\mathcal{Z}$. The $\operatorname{NCO}(2)$ at $\eta=0$ and (5) at $\eta=0_{+}$read, respectively,

$$
\begin{aligned}
\mathbf{0} & =\frac{\partial J}{\partial \mathbf{u}}\left(\mathbf{u}^{*}, \boldsymbol{\theta}^{*}\right)+\boldsymbol{\lambda}_{\mathcal{I}^{*}}^{*} \frac{\partial \mathbf{G}_{\mathcal{I}^{*}}}{\partial \mathbf{u}}\left(\mathbf{u}^{*}, \boldsymbol{\theta}^{*}\right), \\
\mathbf{0} & =\left.\frac{\partial J}{\partial \mathbf{u}}(\tilde{\mathbf{u}}, \tilde{\boldsymbol{\theta}})\right|_{\eta=0_{+}}+\left.\tilde{\boldsymbol{\lambda}}_{\tilde{\mathcal{I}}}^{T}\left(\eta=0_{+}\right) \frac{\partial \mathbf{G}_{\tilde{\mathcal{I}}}}{\partial \mathbf{u}}(\tilde{\mathbf{u}}, \tilde{\boldsymbol{\theta}})\right|_{\eta=0_{+}} .
\end{aligned}
$$

The continuity of the derivatives of $J$ and $\mathbf{G}$ w.r.t. $\mathbf{u}$ and $\boldsymbol{\theta}$, and of the latter two w.r.t. $\eta$ implies 


$$
\begin{aligned}
& \left.\frac{\partial J}{\partial \mathbf{u}}(\tilde{\mathbf{u}}, \tilde{\boldsymbol{\theta}})\right|_{\eta=0_{+}}=\frac{\partial J}{\partial \mathbf{u}}\left(\mathbf{u}^{*}, \boldsymbol{\theta}^{*}\right), \\
& \left.\frac{\partial \mathbf{G}_{\tilde{\mathcal{I}}}}{\partial \mathbf{u}}(\tilde{\mathbf{u}}, \tilde{\boldsymbol{\theta}})\right|_{\eta=0_{+}}=\frac{\partial \mathbf{G}_{\mathcal{I}^{*}}}{\partial \mathbf{u}}\left(\mathbf{u}^{*}, \boldsymbol{\theta}^{*}\right) .
\end{aligned}
$$

Using the last relations, (A.1) yields

$$
\begin{aligned}
\mathbf{0}=\left\{\boldsymbol{\lambda}_{\tilde{\mathcal{I}}}^{*}-\tilde{\boldsymbol{\lambda}}_{\tilde{\mathcal{I}}}(\eta=\right. & \left.\left.0_{+}\right)\right\}^{T} \frac{\partial \mathbf{G}_{\tilde{\mathcal{I}}}}{\partial \mathbf{u}}\left(\mathbf{u}^{*}, \boldsymbol{\theta}^{*}\right) \\
& +\boldsymbol{\lambda}_{\mathcal{Z}}^{* T} \frac{\partial \mathbf{G}_{\mathcal{Z}}}{\partial \mathbf{u}}\left(\mathbf{u}^{*}, \boldsymbol{\theta}^{*}\right) .
\end{aligned}
$$

The last equation is, however, a contradiction since $\boldsymbol{\lambda}_{\mathcal{Z}}^{*} \neq \mathbf{0}$ by definition of $\mathcal{Z}$, and column vectors of $\frac{\partial \mathbf{G}_{\tilde{\mathcal{I}}}}{\partial \mathbf{u}}\left(\mathbf{u}^{*}, \boldsymbol{\theta}^{*}\right)$ and $\frac{\partial \mathbf{G}_{\mathcal{Z}}}{\partial \mathbf{u}}\left(\mathbf{u}^{*}, \boldsymbol{\theta}^{*}\right)$ are independent by the assumed constraint qualification. Hence, $\mathcal{I}^{*} \supset \tilde{\mathcal{I}}_{+}$is impossible.

Step 3: (Impossibility of Replacement in Strict Active Set) Let us assume the contrary, viz., only a part of $\mathcal{I}^{*}$ belongs to $\tilde{\mathcal{I}}_{+}$while there are some new indices in the latter that are not present in $\mathcal{I}^{*}$. That is,

$$
\mathcal{I}^{*}=\mathcal{C} \cup \mathcal{Z}_{1}, \quad \tilde{\mathcal{I}}_{+}=\mathcal{C} \cup \mathcal{Z}_{2}, \quad \mathcal{C} \cap \mathcal{Z}_{1} \cap \mathcal{Z}_{2}=\emptyset .
$$

Thus, this case is a combination of decrease by some indices and increase by some other indices of $\mathcal{I}^{*}$. Following a line of reasoning similar to that of Step 2, it can be shown that this leads to contradictions. Thus, replacement of constraints in the strict active set is also impossible.

Hence, the only possibility that remains is $\mathcal{I}^{*} \subseteq \tilde{\mathcal{I}}_{+}$. Moreover, from the result of Step 1, it is evident that $\tilde{\mathcal{I}}_{+} \backslash \mathcal{I}^{*} \subseteq \mathcal{J}^{*}$.

The same arguments can be applied for the case $\eta=0_{-}$ and so the same result holds for $\tilde{\mathcal{I}}_{-}$too.

Proof of Lemma 3: Continuity of the Lagrange multipliers corresponding to marginally active and inactive constraints is trivial since they stay at zero. To compute $\tilde{\boldsymbol{\lambda}}_{\tilde{\mathcal{I}}}$ at $\eta=0_{+}$, recall from the proof of Lemma 2 that the system of equations (A.1) holds at $\eta=0$ and $0_{+}$, respectively.

Since Lemma 1 holds, results of Lemma 2 hold. Hence, using the fact that $\tilde{\mathcal{I}}_{+}=\mathcal{I}^{*} \cup \mathcal{L}, \mathcal{L} \subseteq \mathcal{J}^{*}$, the system above leads to

$$
\begin{array}{r}
\mathbf{0}=\left\{\boldsymbol{\lambda}_{\mathcal{I}^{*}}^{*}-\tilde{\boldsymbol{\lambda}}_{\mathcal{I}^{*}}\left(\eta=0_{+}\right)\right\}^{T} \frac{\partial \mathbf{G}_{\mathcal{I}^{*}}}{\partial \mathbf{u}}\left(\mathbf{u}^{*}, \boldsymbol{\theta}^{*}\right) \\
+\tilde{\boldsymbol{\lambda}}_{\mathcal{L}}\left(\eta=0_{+}\right)^{T} \frac{\partial \mathbf{G}_{\mathcal{L}}}{\partial \mathbf{u}}\left(\mathbf{u}^{*}, \boldsymbol{\theta}^{*}\right) .
\end{array}
$$

Since $\frac{\partial \mathbf{G}_{\mathcal{I}^{*} \cup \mathcal{J}^{*}}}{\partial \mathbf{u}}\left(\mathbf{u}^{*}, \boldsymbol{\theta}^{*}\right)$ is full rank due to constraint qualification assumption, column vectors of $\frac{\partial \mathbf{G}_{\mathcal{I}^{*}}}{\partial \mathbf{u}}\left(\mathbf{u}^{*}, \boldsymbol{\theta}^{*}\right)$ and $\frac{\partial \mathbf{G}_{\mathcal{L}}}{\partial \mathbf{u}}\left(\mathbf{u}^{*}, \boldsymbol{\theta}^{*}\right)$ are independent. Hence, it must hold that

In summary,

$$
\begin{aligned}
\boldsymbol{\lambda}_{\mathcal{I}^{*}}^{*}-\tilde{\boldsymbol{\lambda}}_{\mathcal{I}^{*}}\left(\eta=0_{+}\right) & =\mathbf{0}, \\
\tilde{\boldsymbol{\lambda}}_{\mathcal{L}}\left(\eta=0_{+}\right) & =\mathbf{0}=\boldsymbol{\lambda}_{\mathcal{L}}^{*} .
\end{aligned}
$$

$$
\tilde{\boldsymbol{\lambda}}_{\mathcal{I}^{*} \cup \mathcal{L}}\left(\eta=0_{+}\right)=\tilde{\boldsymbol{\lambda}}_{\tilde{\mathcal{I}}}\left(\eta=0_{+}\right)=\boldsymbol{\lambda}_{\tilde{\mathcal{I}}}^{*} .
$$

A similar argument can be put forward for $\eta=0_{-}$. Hence, $\tilde{\boldsymbol{\lambda}}_{\tilde{\mathcal{I}}}(\eta)$ is continuous at $\eta=0$, and so, as in Lemma 1 , we have

$$
\tilde{\boldsymbol{\lambda}}_{\tilde{\mathcal{I}}}(\eta)-\boldsymbol{\lambda}_{\tilde{\mathcal{I}}}^{*}=O(\eta) .
$$

\section{Appendix B. LEGEND FOR THE CONTOUR PLOTS}

The legend for all contour plots is as follows:

- Curves in magenta : iso-cost contours (at perturbed parameters)

- Blue dashed line : $G_{1}\left(\mathbf{u}, \boldsymbol{\theta}_{0}\right)=0$ curve

- Red dashed line : $G_{1}(\mathbf{u}, \tilde{\boldsymbol{\theta}})=0$ curve

- Blue line : $G_{2}\left(\mathbf{u}, \boldsymbol{\theta}_{0}\right)=0$ curve

- Red line : $G_{2}(\mathbf{u}, \tilde{\boldsymbol{\theta}})=0$ curve

- Blue arrow : direction of $\mathbf{J}_{\mathbf{u}}$ at nominal optimal solution

- Black arrow : direction of $\mathbf{J}_{\mathbf{u}}$ at solution of modifier adaptation

- Red arrow : direction of $\mathbf{J}_{\mathbf{u}}$ at perturbed optimal solution

- Origin of blue arrow (blue $\diamond)$ : nominal optimal solution

- Origin of black arrow (black $\triangle$ ) : solution of modifier adaptation

- Origin of red arrow $($ red $*)$ : perturbed optimal solution 